\title{
A comparison between OFDM and single-carrier with cyclic prefix using channel coding and frequency-selective block fading channels
}

\author{
Amanda de Paula, Cristiano Panazio
}

\begin{abstract}
This article aims to establish a performance comparison between the single-carrier with cyclic prefix (SCCP) and the orthogonal frequency division multiplexing (OFDM) for frequency selective block fading channels without channel information on the transmitter side and using error correcting codes. For the SCCP scheme, it will be considered both linear and decision-feedback equalizers implementations. We will assess both schemes bit-error rates and capacities for different modulations and coding rates using different approaches. Firstly, analytical results are obtained for a convex analysis framework based on the OFDM effective signal-to-noise ratio and the cutoff rate together with the Shannon capacity analysis. In addition, Monte Carlo simulations are evaluated, corroborating previous analytical results and giving further insights on the comparison.
\end{abstract}

Index Terms-OFDM, single-carrier, frequency domain equalization, decision feedback equalizer, channel coding, channel capacity.

\section{INTRODUCTION}

$\mathbf{T}$ HE orthogonal frequency division multiplexing (OFDM) [1] has become a popular transmission technique for frequency selective channels in which digital signals are transmitted through orthogonal subcarriers. Despite its popularity, the OFDM presents some drawbacks. In particular, it heavily depends on channel coding to achieve good performance by exploiting channel frequency diversity [2], [3]. This comes from the fact that if the data carried on an attenuated subcarrier is lost, the coded OFDM relies on other non-faded subcarriers to be recover it. However, the single-carrier is able to exploit such diversity even in the absence of channel coding, since each transmitted symbol spreads throughout the entire used band due to its much smaller symbol duration as compared to the OFDM. Furthermore, the cyclic-prefix (CP) and the one-tap equalizer techniques [4], which allow low complexity equalization, are not a privilege of the OFDM and they can also be applied to the single-carrier, giving rise to the so-called single-carrier with cyclic-prefix (SCCP). However, despite some similarities between both modulation techniques, the characteristics of the received signal differ significantly and may lead to different performances when used in the same channel. For instance, in addition to Gaussian noise, the received signal of the SCCP is corrupted by intersymbol interference (ISI), whereas the OFDM signal is just a rotated and scaled version of the transmitted signal plus Gaussian noise.

A. Paula and C. Panazio are with the Escola Politécnica, University of São Paulo, Av. Prof. Luciano Gualberto, tr. 3, 158, São Paulo-SP, Brazil, tel.: (+55) (11) 30919797 e-mail: \{amanda,cpanazio\}@1cs.poli.usp.br
Therefore, the equalizer in the SCCP has to sub-optimally mitigate ISI by using, for example, a minimum mean square error (MMSE) criterion, and the one-tap equalizer in the OFDM just has to provide gain and phase corrections, without any performance loss. Furthermore, since each subcarrier is subject to a different phase and gain, the OFDM symbols that go to the channel decoder are subject to different signalto-noise ratios (SNRs), which is analogous to a time-varying flat fading channel, while the SCCP averages the signal over all subcarriers. Since the same error correcting code performs differently in both cases, we should expect some performance differences. Therefore, a natural question arises: how do their performances compare?

In order to answer that question, several comparisons have been published. We can separate them into two groups: one group assumes that the channel gains are known on the transmitter side and another one where they are unknown. In the first group, the OFDM can easily optimize its capacity by performing water-filling/bit-loading [5], which basically equalizes the signal-to-noise ratio per bit $\left(E_{b} / N_{0}\right)$ in the active subcarriers, eliminating the OFDM problems reported in the previous paragraph, whereas the SCCP, with a linear equalizer (LE), can also provide a capacity gain, but in a less extent [6]-[8]. When a decision-feedback equalizer (DFE) [9] is employed in the single carrier, it can hypothetically achieve capacity gains similar to that of the OFDM, [7], [10], or if Tomlisom-Harashima DFE [11] is used [12], [13], the optimal capacity can be achieved, but at the expense of much higher complexity. Such results do not leave space for many additional considerations or discussions and, hence, we are not going to develop them here.

However, in the second group, in which no optimization is performed on the transmitter side, all the problems discussed at the beginning of this section make the differences between both schemes much more subtle. Our main objective in this paper is to show some established results and provide new ones to well clarify some of the differences between them. In this context, there are many works, such as [2], [10], [14][20]. Some of them [2], [14], [17] are restricted to Monte Carlo simulations that naturally hinders the extent of any conclusion, and fails to provide comprehensive scenarios. For instance, they are restricted to just one kind of modulation, such as binary phase-shift keying or quadrature phase-shift keying (QPSK), others do not take into account the frequency domain DFE that may provide better performance than the LE with little additional complexity and finally, they fail to 
generalize the comparison to any channel configuration.

Other comparisons establish an analytical treatment to the problem, but their results are not applied to schemes using channel coding [15], [19], [20]. Concerning the comparison under a coded context, [16] provides an interesting analytical result using the cutoff rate [21], but it solely analyzes the effect of the coding rate considering linear equalization for the SCCP and a single channel configuration scenario. Furthermore, it is subject to an unrealistic assumption of infinite code length that analyzes solely the channel capacity. In [3] and [10] the coded case is also analyzed, but their results also depend on the infinite code lengths hypothesis.

In this paper, we aim to revisit some of the results commented above, but also to provide new general results and additional insights in order to complement these comparisons. Using a convex analysis framework, some analytical results can be provided when used together with the concept of effective signal-to-noise ratio $\left(S N R_{e f f}\right)$ for OFDM [22] or the cutoff rate. Moreover, additional insights on the comparison are presented by using the Shannon capacity and Monte Carlo simulations, which also corroborate the analytical results.

This paper is organized as follows. In Section II, the system model to be considered throughout this work is described. Section III revisits some results provided for the uncoded case. In Section IV the comparison is accomplished in the coded case using three different approaches: the effective SNR, capacity comparison and Monte Carlo simulation. Conclusions are stated in Section V.

\section{SySTEM MODEL}

The similarities between the OFDM and the SCCP allow us to describe both modulation schemes in a unified system model [15] depicted in Fig. 1. The two modulation schemes are distinguished by the linear precoding matrix $\mathcal{P}$. In the OFDM case, the transmitted symbols are obtained from the inverse fast Fourier transform (IFFT) of the data vector $\mathbf{X}$, thus the data vector is not pre-processed and the transformation $\mathcal{P}$ is replaced by the identity matrix. On the other hand, in the SCCP scheme, the vector of symbols itself is transmitted. In order to accomplish that, the precoding matrix $\mathcal{P}$ must be replaced by the Fourier matrix. After the IFFT, a CP is inserted

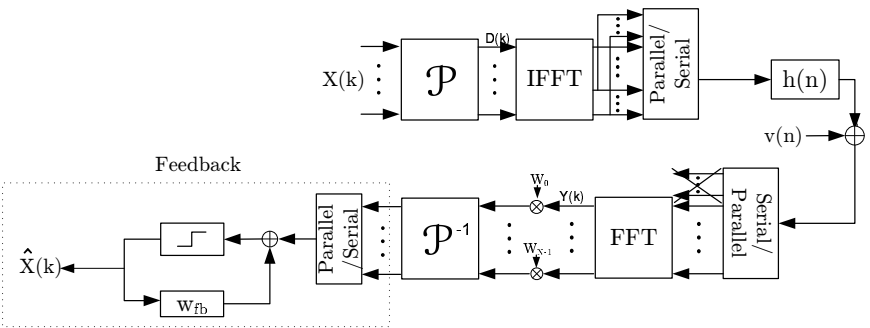

Fig. 1. Unified model, where the feedback filter is appended to implement a decision feedback equalizer for the SCCP.

that allows us to equalize the received signal in the frequency domain with a simple one-tap equalizer [4], since it eliminates the interblock interference and, in time-invariant channels, it keeps the orthogonality between subcarriers. In the OFDM, where there is no ISI, the equalization is reduced to phase and magnitude compensations in each subcarrier by the onetap equalizer, without introducing any performance penalty for the signal decoding process. By contrast, the SCCP can also use the one-tap equalizer to perform linear equalization, but due to the presence of ISI, it cannot optimally mitigate ISI in the presence of noise without introducing a large performance penalty due to noise enhancement. A good compromise in this case is to use the minimum mean square error (MMSE) criterion [23], which will be adopted herein.

Besides the LE, the SCCP allows us to use a nonlinear equalization scheme to better mitigate the ISI when noise is present. One of such schemes is the DFE. As illustrated in Fig. 1 , the feedforward filtering is accomplished in the frequency domain using the one-tap equalizer and the feedback filtering is accomplished in the time domain. Applying the MMSE criterion, the feedback and feedforward filters coefficients can be calculated as shown in [10]. Optimal performance is achieved when the number of DFE feedback coefficients is equal to the channel length minus one [24]. When the error propagation effect is not taken into account, the DFE is said to be perfect. This assumption is important in order to make the DFE mathematical analysis more feasible.

The DFE-SCCP poses a problem in the initialization of the feedback filter, since it requires the access to the last symbols of the block, which have not yet been processed. An alternative to overcome this issue is to implement the DFE with the unique word (UW) technique [25]. With such approach, the same rate or efficiency as the one obtained with the $\mathrm{CP}$ can be attained, but a larger fast Fourier transform (FFT) is required. If the FFT length is the same for both schemes, the rates are different. However, if the block size is large compared to the CP or UW lengths, the difference between both techniques are insignificant. Hence, in order to simplify the analysis, we will consider the DFE-SCCP and assume that the last symbols of the block are known and are used to initialize the feedback filter.

For all simulations, we have used $N=512$ subcarriers in the OFDM, as well considered $N=512$ symbols in the SCCP block. The $\mathrm{CP}$ length was the minimum required to perform the equalization with the one-tap equalizer, i.e., the channel length minus one.

\section{UNCODED SCENARIO}

In this section, the two schemes will be compared considering that the transmission is accomplished without channel coding. In an additive white Gaussian noise (AWGN) scenario and considering a $\mathrm{M}$-ary quadrature amplitude modulation (MQAM), the bit-error rate (BER) can be expressed as

$$
\mathrm{BER}=\alpha Q(\sqrt{\beta \mathrm{SNR}}),
$$

where $Q(x)$ is the complementary error function, $\alpha$ and $\beta$ are constants given by

$$
\alpha=\frac{4}{\log _{2}(M)}\left(1-\frac{1}{\sqrt{M}}\right) \quad \beta=\frac{3}{M-1}
$$

and the SNR is the received signal-to-noise ratio.

In the SCCP scheme, when the transmission is accomplished through a frequency selective channel, and considering that the 
residual ISI can be modeled as a Gaussian random variable, the system BER can be expressed similarly to (1)

$$
\mathrm{BER}=\alpha Q\left(\sqrt{\beta \mathrm{SNR}_{\mathrm{eq}}}\right),
$$

where $\mathrm{SNR}_{\mathrm{eq}}$ is the residual SNR after the equalization process. The $\mathrm{SNR}_{\mathrm{eq}}$ will depend on the type of equalizer chosen for the single-carrier. Throughout this paper, we denote the $\mathrm{SNR}_{\mathrm{eq}}$ in the LE output as $\mathrm{SNR}_{\mathrm{LE}}$ and the $\mathrm{SNR}_{\mathrm{eq}}$ in the DFE output as $\mathrm{SNR}_{\mathrm{DFE}}$.

The $\mathrm{SNR}_{\mathrm{LE}}$ is given by [23]

$$
\mathrm{SNR}_{\mathrm{LE}}=\frac{\sum_{k=0}^{N-1} \frac{\gamma\left|H_{k}\right|^{2}}{1+\gamma\left|H_{k}\right|^{2}}}{\sum_{k=0}^{N-1} \frac{1}{1+\gamma\left|H_{k}\right|^{2}}},
$$

where $\gamma$ is the average SNR, $\gamma=\frac{\sigma_{X}^{2}}{\sigma_{v}^{2}}, \sigma_{X}^{2}$ is the average symbol power, $\sigma_{v}^{2}$ is the noise variance, $H_{k}$ is the $k^{t h}$-channel frequency response component and $N$ is the length of the single-carrier block. This expression can be written as:

$$
\operatorname{SNR}_{\mathrm{LE}}=\text { harmmean }\left\{1+\gamma|\mathbf{H}|^{2}\right\}-1,
$$

where harmmean $\{\cdot\}$ is the harmonic mean operator [26].

Considering the perfect DFE equalizer, the $\mathrm{SNR}_{\mathrm{DFE}}$ is given by [27]

$$
\mathrm{SNR}_{\mathrm{DFE}}=\exp \left\{\frac{1}{N} \sum_{k=0}^{N-1} \log \left(1+\gamma\left|H_{k}\right|^{2}\right)\right\}-1 .
$$

This expression can also be rewritten in terms of the geometric mean operator

$$
\operatorname{SNR}_{\mathrm{DFE}}=\text { geomean }\left\{1+\gamma|\mathbf{H}|^{2}\right\}-1 .
$$

The geometric mean is known to be always equal to or greater than the harmonic mean [26]. Therefore, $\mathrm{SNR}_{\mathrm{DFE}} \geq$ $\mathrm{SNR}_{\mathrm{LE}}$. Given the monotonically decreasing behavior of the $Q(x)$ function, this fact asserts that the LE cannot surpass the DFE performance in terms of BER.

In order to derive the OFDM BER, we emphasize that, in the uncoded scenario, we can state that there is an independency relation among the messages transmitted in each subcarrier. Thence, the OFDM mean BER can be expressed as the mean of the BER in each individual subcarrier

$$
\mathrm{BER}_{\mathrm{OFDM}}=\frac{1}{N} \sum_{k=0}^{N-1} \alpha Q\left(\sqrt{\beta \mathrm{SNR}_{k}}\right),
$$

where $\mathrm{SNR}_{k}$ is the $\mathrm{SNR}$ in the $k^{t h}-$ subcarrier, given by

$$
\mathrm{SNR}_{k}=\gamma\left|H_{k}\right|^{2} \text {. }
$$

In [15], the BER of the OFDM given by (8) and the BER of the LE-SCCP were compared and it was stated that, for QPSK modulation, the LE-SCCP outperforms the OFDM. In [28], the BER of the OFDM was compared to the BER of the DFE-SCCP and it was proved that the DFE-SCCP surpasses the OFDM for QPSK and 16-QAM modulations for any channel configuration. In [15] and [28] the schemes are compared through a simple convex analysis. This analysis can be extended for higher order modulation schemes and in those cases it is noted that the problem is not convex anymore and thereby the OFDM can excel the DFE-SCCP in some channel configurations.

Furthermore, from the results already provided in the literature, we can conclude that the SCCP is a better option in comparison to the OFDM when lower order modulations are employed. The question that arises is if this relation holds when the transmission is accomplished with channel coding.

\section{CODED SCENARIO}

As pointed out earlier, the uncoded comparison between the OFDM and SCCP schemes is analytically tractable and it is possible to attain closed-form results stating which one provides a better performance in terms of the BER. In the coded scenario, however, the comparison is not straightforward due to two main reasons. The first one is that it may not be simple to derive the code BER function. The second one, and more restrictive condition, is that there is not an independency relation among the information transmitted in the OFDM subcarriers anymore. Therefore, we cannot state that the OFDM BER equals the mean of the BER in the subcarriers.

There are some alternatives to overcome these difficulties in the coded case comparison: a) analyzing the OFDM by the means of the effective SNR, b) capacity analysis and c) Monte Carlo simulation.

These three alternatives present advantages and drawbacks. As will be detailed in the next subsection, the effective SNR provides an analytical treatment to the comparison. However, the analysis with this tool is not very conclusive. The capacity analysis also allows an analytical treatment to the problem, but on the other hand, one must consider some unrealistic assumptions. Finally, the Monte Carlo simulation provides interesting insights about the comparison regarding important parameters such as coding rate and modulation schemes, although its results cannot be rigorously generalized.

Throughout this paper, these three different approaches will be exploited in order to draw some interesting conclusions concerning the comparison between the transmissions schemes. In the next subsection, we analyze the OFDM with the effective SNR tool.

\section{A. Effective SNR}

The effective SNR [22], [29]-[32], $\mathrm{SNR}_{\text {eff }}$, is a figure of merit of the OFDM scheme applied for block fading channels that maps the SNR for each subcarrier in a single SNR value that corresponds to the SNR of an AWGN channel.

Among the different types of effective SNR mappings, the exponential effective SNR is the most commonly used:

$$
\mathrm{SNR}_{\mathrm{eff}}=-\lambda \log \left(\frac{1}{N} \sum_{k=0}^{N-1} \exp \left(-\gamma \frac{\left|H_{k}\right|^{2}}{\lambda}\right)\right),
$$

where $\lambda$ is a positive parameter that depends on the channel coding scheme and the modulation order.

By using the $\mathrm{SNR}_{\text {eff }}$, the BER of the OFDM is expressed by

$$
\mathrm{BER}_{\mathrm{OFDM}}=\alpha Q_{c}\left(\sqrt{\beta \mathrm{SNR}_{\mathrm{eff}}}\right),
$$


where $Q_{c}(x)$ is the error function associated to the applied error correcting code.

Concerning the LE-SCCP and the DFE-SCCP, the BER can be expressed as

$$
\begin{gathered}
\mathrm{BER}_{\mathrm{LE}}=\alpha Q_{c}\left(\sqrt{\beta \mathrm{SNR}_{\mathrm{LE}}}\right), \\
\mathrm{BER}_{\mathrm{DFE}}=\alpha Q_{c}\left(\sqrt{\beta \mathrm{SNR}_{\mathrm{DFE}}}\right) .
\end{gathered}
$$

Since the error function $Q_{c}(x)$ is the same for both schemes, and it is a monotonically decreasing function, it suffices to compare their respective SNR values. In order to do so, we proceed similarly to [28] and define the following auxiliary functions:

$$
\begin{array}{r}
\tau(x)=\exp \left(\frac{-x}{\lambda}\right), \\
\theta(x)=\frac{1}{1+x}, \\
\phi(x)=\log (x+1) .
\end{array}
$$

Applying these auxiliary functions to the SNR expressions, our task is simplified to compare theses relations:

$$
\begin{aligned}
\mathrm{SNR}_{\mathrm{eff}} & =\tau^{-1}\left(\frac{1}{N} \sum_{k=0}^{N-1} \tau\left(\gamma\left|H_{k}\right|^{2}\right)\right), \\
\mathrm{SNR}_{\mathrm{LE}} & =\theta^{-1}\left(\frac{1}{N} \sum_{k=0}^{N-1} \theta\left(\gamma\left|H_{k}\right|^{2}\right)\right), \\
\mathrm{SNR}_{\mathrm{DFE}} & =\phi^{-1}\left(\frac{1}{N} \sum_{k=0}^{N-1} \phi\left(\gamma\left|H_{k}\right|^{2}\right)\right) .
\end{aligned}
$$

It has already been stated that $\mathrm{SNR}_{\mathrm{DFE}} \geq \mathrm{SNR}_{\mathrm{LE}}$. Therefore, our remaining tasks are to compare $\mathrm{SNR}_{\mathrm{LE}}$ and $\mathrm{SNR}_{\mathrm{eff}}$ as well as to compare $\mathrm{SNR}_{\mathrm{DFE}}$ and $\mathrm{SNR}_{\text {eff }}$.

Firstly, $\mathrm{SNR}_{\mathrm{LE}}$ and $\mathrm{SNR}_{\text {eff }}$ are compared. In order to do so, the function $\tau(x)$ is applied to (15) and (16):

$$
\begin{aligned}
\tau\left(\mathrm{SNR}_{\text {eff }}\right) & =\frac{1}{N} \sum_{k=0}^{N-1} \tau\left(\gamma\left|H_{k}\right|^{2}\right), \\
\tau\left(\mathrm{SNR}_{\mathrm{LE}}\right) & =\tau\left(\theta^{-1}\left(\frac{1}{N} \sum_{k=0}^{N-1} \theta\left(\gamma\left|H_{k}\right|^{2}\right)\right)\right) .
\end{aligned}
$$

We can also define

$$
\psi(x)=\tau\left(\theta^{-1}(x)\right) \Leftrightarrow \tau(x)=\psi(\theta(x)) .
$$

Rewriting (18) and (19) in terms of $\psi(x)$, we have that:

$$
\begin{aligned}
\tau\left(\mathrm{SNR}_{\mathrm{eff}}\right) & =\frac{1}{N} \sum_{k=0}^{N-1} \psi\left(\theta\left(\gamma\left|H_{k}\right|^{2}\right)\right), \\
\tau\left(\mathrm{SNR}_{\mathrm{LE}}\right) & =\psi\left(\frac{1}{N} \sum_{k=0}^{N-1} \theta\left(\gamma\left|H_{k}\right|^{2}\right)\right) .
\end{aligned}
$$

Exploiting the fact that the function $\tau(x)$ is strictly decreasing, the functions $\mathrm{SNR}_{\mathrm{LE}}$ and $\mathrm{SNR}_{\text {eff }}$ can be compared by means of the comparison between (21) and (22). If $\tau\left(\mathrm{SNR}_{\mathrm{eff}}\right) \geq \tau\left(\mathrm{SNR}_{\mathrm{LE}}\right)$, we have that $\mathrm{SNR}_{\mathrm{eff}} \leq \mathrm{SNR}_{\mathrm{LE}}$.

The comparison between (21) and (22) can be accomplished by analyzing the convexity of the function $\psi(x)$. The Jensen inequality [26] states that if the function $\psi(x)$ is convex, we have that:

$$
\psi\left(\frac{1}{N} \sum_{k=0}^{N-1} \theta\left(\gamma\left|H_{k}\right|^{2}\right)\right) \leq \frac{1}{N} \sum_{k=0}^{N-1} \psi\left(\theta\left(\gamma\left|H_{k}\right|^{2}\right)\right)
$$

which implies that $\mathrm{SNR}_{\mathrm{eff}} \leq \mathrm{SNR}_{\mathrm{LE}}$.

In order to analyze the convexity of

$$
\psi(x)=\exp \left(-\frac{1}{\lambda}\left(\frac{1}{x}-1\right)\right)
$$

it is necessary to analyze its second derivative:

$$
\frac{d^{2}}{d x^{2}} \psi(x)=\frac{\exp \left(\frac{-1}{\lambda}\left(\frac{1}{x}-1\right)\right)}{\lambda x^{3}}\left(-2+\frac{1}{\lambda x}\right) .
$$
have:

Calculating this second derivative at $x_{k}=\theta\left(\gamma\left|H_{k}\right|^{2}\right)$, we

$$
\begin{gathered}
\left.\frac{d^{2}}{d x^{2}} \psi(x)\right|_{\theta\left(\gamma\left|H_{k}\right|^{2}\right)}=\frac{1}{\lambda}\left(1+\gamma\left|H_{k}\right|^{2}\right)^{3} \\
\times\left[\exp \left(-\frac{\gamma\left|H_{k}\right|^{2}}{\lambda}\right)\left(-2+\frac{1}{\lambda}+\frac{\gamma\left|H_{k}\right|^{2}}{\lambda}\right)\right] .
\end{gathered}
$$

The signal of the second derivative is determined by the third factor, since the other two factors are always positive. If $\lambda \leq \frac{1}{2}$, this factor is positive, and in this special case, the function is convex and it can be stated that the $\mathrm{SNR}_{\text {eff }} \leq$ $\mathrm{SNR}_{\mathrm{LE}}$. However, even if $\lambda \geq \frac{1}{2}$, the function can still be convex if

$$
\gamma \geq \frac{2 \lambda-1}{\left|H_{k}\right|^{2}}
$$

and, in such a case, the condition defined in (27) must hold for all subcarriers $k$. It is worth noting that this situation will be true in the absence of channel spectral nulls $\left(\left|H_{k}\right| \neq 0, \forall k\right)$ and for SNR $\rightarrow \infty$, and in this particular case, we can infer that the LE-SCCP will outperform the OFDM. This result contrasts with the uncoded scenario where the LE-SCCP surpasses the OFDM for any channel configuration if QPSK is employed.

The comparison between the DFE-SCCP and the OFDM can be carried out analogously to the former comparison. Applying the function $\tau(x)$ to (15) and (17), we have that

$$
\begin{aligned}
\tau\left(\mathrm{SNR}_{\mathrm{eff}}\right) & =\frac{1}{N} \sum_{k=0}^{N-1} \tau\left(\gamma\left|H_{k}\right|^{2}\right) \\
\tau\left(\mathrm{SNR}_{\mathrm{DFE}}\right) & =\tau\left(\phi^{-1}\left(\frac{1}{N} \sum_{k=0}^{N-1} \phi\left(\gamma\left|H_{k}\right|^{2}\right)\right)\right)
\end{aligned}
$$

In addition, $\xi(x)=\tau\left(\phi^{-1}(x)\right)$ is defined and (28) and (29) are expressed in terms of $\xi(x)$ :

$$
\begin{aligned}
\tau\left(\mathrm{SNR}_{\mathrm{eff}}\right) & =\frac{1}{N} \sum_{k=0}^{N-1} \xi\left(\phi\left(\gamma\left|H_{k}\right|^{2}\right)\right) \\
\tau\left(\mathrm{SNR}_{\mathrm{DFE}}\right) & =\xi\left(\frac{1}{N} \sum_{k=0}^{N-1} \phi\left(\gamma\left|H_{k}\right|^{2}\right)\right) .
\end{aligned}
$$


Once again, we can analyze the convexity of the function $\xi(x)$ in order to establish a comparison between the two schemes. The function $\xi(x)$ is given by

$$
\xi(x)=\exp \left(-\frac{1}{\lambda}(\exp (x)-1)\right),
$$

and its second derivative can be written as:

$$
\frac{d^{2}}{d x^{2}} \xi(x)=\frac{1}{\lambda} \exp (x) \xi(x)\left(-1+\frac{\exp (x)}{\lambda}\right) .
$$
have:

Calculating this second derivative at $y_{k}=\phi\left(\gamma\left|H_{k}\right|^{2}\right)$, we

$$
\begin{array}{r}
\left.\frac{d^{2}}{d x^{2}} \xi(x)\right|_{\phi\left(\gamma\left|H_{k}\right|^{2}\right)}=\frac{1}{\lambda}\left(1+\gamma\left|H_{k}\right|^{2}\right) \\
\times\left[\exp \left(-\frac{\gamma}{\lambda}\left|H_{k}\right|^{2}\right)\left(-1+\frac{1+\gamma\left|H_{k}\right|^{2}}{\lambda}\right)\right] .
\end{array}
$$

If $\lambda \leq 1$, we conclude that $\left.\frac{d^{2}}{d x^{2}} \xi(x)\right|_{\phi\left(\gamma\left|H_{k}\right|^{2}\right)} \geq 0$ Thus, in this situation, the convexity of the function $\xi(x)$ is guaranteed, which means that the $\mathrm{SNR}_{\text {eff }} \leq \mathrm{SNR}_{\mathrm{DFE}}$ and therefore, $\mathrm{BER}_{\mathrm{DFE}} \leq \mathrm{BER}_{\mathrm{OFDM}}$.

On the other hand, in case $\lambda \geq 1$, the SNR values for which the function $\xi(x)$ is convex are given by

$$
\gamma \geq \frac{\lambda-1}{\left|H_{k}\right|^{2}}
$$

Similarly to the LE-SCCP case, this is achieved only for the absence of spectral nulls and for SNR $\rightarrow \infty$. If all subcarriers satisfy this special condition, the DFE-SCCP will outperform the OFDM. The problem is that for both the LE-SCCP and the DFE-SCCP, the most practical channel coding and modulation schemes lead to $\lambda>1$ [33]-[35]. Therefore, except in the case that $\gamma \rightarrow \infty$, which is not of practical interest, the problem is non-convex and no definitive conclusion can be drawn.

\section{B. Capacity Analysis}

The results obtained with the $\mathrm{SNR}_{\text {eff }}$ indicate that the BER performance of the OFDM is not so different from that presented by the SCCP, contrarily to the uncoded case. In order to deepen the analysis, at the expense of a not-so-practical hypothesis that the code has infinite length, we will show how the schemes capacities compare. In order to do so, assuming that the residual ISI is Gaussian, the SCCP capacity can be expressed as

$$
C=\frac{1}{2} \log _{2}\left(1+\mathrm{SNR}_{\mathrm{eq}}\right) .
$$

Concerning the DFE-SCCP, the SNR is given by (6) and the system capacity is given by:

$$
\begin{aligned}
C_{\mathrm{DFE}} & =\frac{1}{2} \log _{2}\left(1+\exp \left\{\frac{1}{N} \sum_{k=0}^{N-1} \log \left(1+\gamma\left|H_{k}\right|^{2}\right)\right\}-1\right) \\
& =\frac{1}{N} \sum_{k=0}^{N-1} \frac{1}{2} \log _{2}\left(1+\gamma\left|H_{k}\right|^{2}\right)
\end{aligned}
$$

but, since $\gamma\left|H_{k}\right|^{2}$ is the SNR for each subcarrier in the OFDM scheme, this is exactly the ergodic capacity for it [36]. Hence, the OFDM and the perfect DFE-SCCP schemes present the same capacity.

The LE-SCCP has already shown to present a SNR lower than the perfect DFE-SCCP. Since, the capacity is an increasing function of the SNR, we can state that the LE-SCCP presents a lower capacity when compared to the perfect DFESCCP and to the OFDM.

The analysis above assumes that Gaussian symbols are transmitted, which is not the case in practice. For ordinary modulations, the capacity can still be numerically evaluated using [37]

$C^{\mathrm{M}-\mathrm{QAM}}(\mathrm{SNR})=-\int_{-\infty}^{+\infty} f_{Y}(y) \log _{2}\left(f_{Y}(y)\right) d y-\frac{1}{2} \log _{2}\left(\underset{(38)}{\frac{2 \pi e}{\mathrm{SNR}}}\right)$

where $f_{Y}(y)$ is the probability density function associated to the received symbol and depends on the modulation order $M$.

Equation (38) has a shape similar to the Gaussian capacity for increasing modulation order and SNR values, i.e., for $M \rightarrow$ $\infty$, with $S N R \rightarrow \infty$. In these conditions, the channel capacity given by (38) presents a performance gap when compared to the Gaussian capacity. That performance gap is known as the shaping gain and is equal to $1.53 \mathrm{~dB}$ [23]. However, when $M$ is finite, it will saturate in $\log _{2}(M)$. This saturation is the reason for the OFDM to present a capacity degradation when compared to the DFE-SCCP, under certain values of SNR. In order words, the OFDM is underusing the capacity of the best subcarriers, which incurs into a capacity loss.

In order to show this, let us consider that the capacity of the SCCP can be evaluated by applying the $\mathrm{SNR}_{\text {eq }}$ in (38):

$$
C_{\mathrm{SCCP}}^{\mathrm{M}-\mathrm{QAM}}=C^{\mathrm{M}-\mathrm{QAM}}\left(\mathrm{SNR}_{\mathrm{eq}}\right) \text {. }
$$

On the other hand, the OFDM ergodic capacity is the average of the capacities in the different subcarriers:

$$
C_{\text {OFDM }}^{\mathrm{M}-\mathrm{QAM}}=\frac{1}{N} \sum_{k=0}^{N-1} C^{\mathrm{M} \text {-QAM }}\left(\gamma\left|H_{k}\right|^{2}\right) .
$$

Consider first that we have $\gamma \rightarrow \infty$, then (39) and (40) converges to $\log _{2}(M)$. If $\gamma$ is small enough to have $C^{\mathrm{M}-\mathrm{QAM}}\left(\gamma\left|H_{k}\right|^{2}\right) \approx C^{\mathrm{M}^{\prime}-\mathrm{QAM}}\left(\gamma\left|H_{k}\right|^{2}\right)$, with $M^{\prime} \gg$ $M, \forall k$ then the capacities are also equal. However, if $C^{\mathrm{M}-\mathrm{QAM}}\left(\gamma\left|H_{k}\right|^{2}\right)$ falls close to the saturation region of (38) for certain values of $k$, then the capacity of the OFDM will be inferior to that of the DFE-SCCP. As an example, the OFDM and the SCCP capacities will be calculated for the unitary norm channel with zeros in $0.95 \exp ( \pm j 0.9 \pi)$, considering 16-QAM modulation, $\gamma=6 \mathrm{~dB}$, and $N=8$ subcarriers or symbols. The results are depicted in Fig. 2. As can be seen, the capacities are almost the same in this case. In addition to the 16-QAM, the 64-QAM capacity curve is also shown in Fig. 2. Considering this modulation, the capacity for both schemes remains practically the same. However, for a higher value of $\gamma$ $(\gamma=11 \mathrm{~dB})$, as shown in Fig. 3, certain values of $\gamma\left|H_{k}\right|^{2}$ fall close to the saturation region generated by (38) which clearly leads to a capacity difference between the OFDM and the DFE-SCCP. Additionally, Fig. 3 also shows that for 64-QAM and the same $\gamma$ that both schemes have the same capacity, since we fall in the case of Fig. 2. 
From the results discussed in the previous paragraph, we can predict that larger deviations of $\left|H_{k}\right|^{2}$ lead to larger differences between the capacities of the OFDM and the DFE-SCCP.

These results will also reflect on the outage probability. For a fixed modulation order, when the coding rate $r \rightarrow 1$, the SNR values approach the saturation region, resulting in higher outage probability to the OFDM when compared to the DFESCCP. Since such results are obtained through semianalytical methods and depend on the channel configuration, they will only be shown in the next section.

In spite of the interesting results obtained through the capacity analysis, the problem cannot be analytically treated. In order to overcome this, the comparison can be performed in terms of the cutoff rate [21], which is a lower bound of the Shannon capacity.

In [16], there is a comparison between the LE-SCCP and the OFDM restricting the analysis to a two-path Rayleigh fading scenario. Next, the conclusions will be extended to an arbitrary channel configuration and to the DFE-SCCP. Firstly, the cutoff rate expressions for the OFDM and the SCCP [16] will be presented and, later, the convex analysis that allow generalizing the behavior of the OFDM and the SCCP with regard to the coding rate for any channel will be provided.

The cutoff rate for the $M$-ary modulation and AWGN scenario can be defined as [21]

$$
R_{0}=-\log _{2}\left(\frac{1}{M^{2}} \sum_{l=0}^{M-1} \sum_{m=0}^{M-1} \mathbb{P}\left\{X_{l} \mapsto X_{m}\right\}\right)
$$

where $\mathbb{P}\left\{X_{l} \mapsto X_{m}\right\}$ represents the pairwise error probability, i.e., the probability of decoding a symbol as $X_{m}$ given that $X_{l}$ has been transmitted.

Considering QPSK modulation, the pairwise error probability is given by

$$
\mathbb{P}\left\{X_{l} \mapsto X_{m}\right\}=Q\left(\sqrt{\frac{\left\|X_{l}-X_{m}\right\|^{2}}{2 \sigma_{v}^{2}}}\right) .
$$

Applying the Chernoff bound, the cutoff rate can be approx-

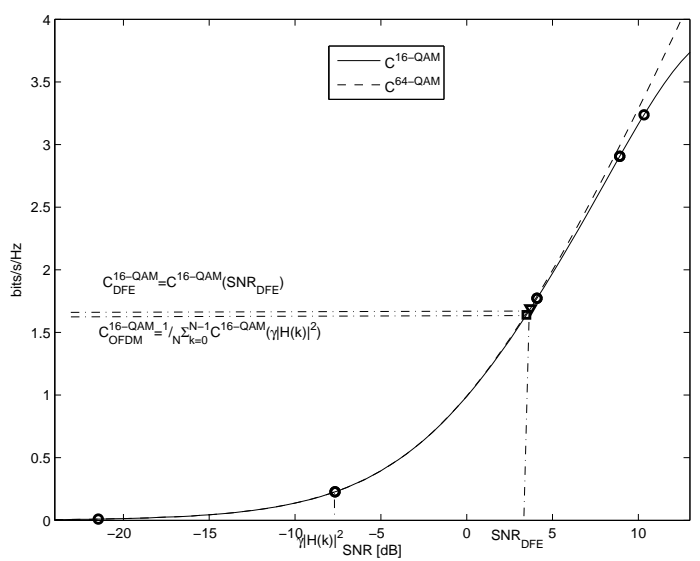

Fig. 2. Capacity for the OFDM and DFE-SCCP schemes considering 16QAM and 64-QAM for a low SNR value.

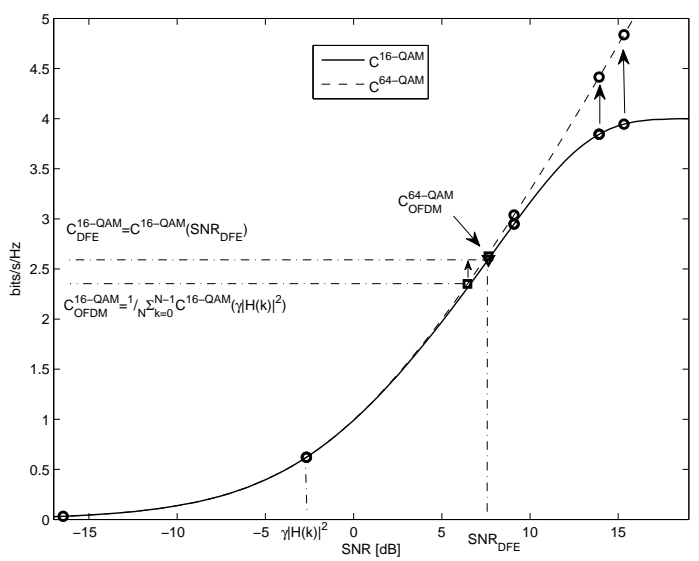

Fig. 3. Capacity for the OFDM and DFE-SCCP schemes considering 16QAM and 64-QAM for a high SNR value.

imated by

$$
R_{0, A W G N}=-\log _{2}\left(\frac{1}{4} \sum_{l=0}^{1} \sum_{m=0}^{1} \exp \left\{-\frac{\left\|X_{l}-X_{m}\right\|^{2}}{4 \sigma_{v}^{2}}\right\}\right),
$$

the metric $\left\|X_{l}-X_{m}\right\|$ represents the Euclidian distance between the symbols $X_{l}$ and $X_{m}$. Defining

$$
A_{l, m}=\frac{\left\|X_{l}-X_{m}\right\|^{2}}{4 \sigma_{X}^{2}}
$$

the cutoff rate can be rewritten as

$$
R_{0, A W G N}=-\log _{2}\left(\frac{1}{4} \sum_{l=0}^{1} \sum_{m=0}^{1} \exp \left\{-A_{l, m} \gamma\right\}\right) .
$$

and invoking ergodicity, the OFDM cutoff rate is expressed as

$R_{0, \text { OFDM }}=-\log _{2}\left(\frac{1}{4} \sum_{l=0}^{1} \sum_{m=0}^{1} \frac{1}{N} \sum_{k=0}^{N-1} \exp \left(-\gamma A_{l, m}\left|H_{k}\right|^{2}\right)\right)$.

Concerning the SCCP and considering that any residual intersymbol interference after equalization can be modeled as a Gaussian random variable, the cutoff rate of the SCCP scheme is

$$
R_{0, S C C P}=-\log _{2}\left(\frac{1}{4} \sum_{l=0}^{1} \sum_{m=0}^{1} \exp \left\{-A_{l, m} \mathrm{SNR}_{\mathrm{eq}}\right\}\right) .
$$

The SNR in the perfect DFE output is known to be greater or equal to the SNR in the LE output. Thus, from (47), we can conclude that the cutoff rate associated to the DFE is greater or equal to the cutoff rate associated to the LE.

Defining the following relations:

$$
\begin{array}{r}
\zeta_{O F D M}=\frac{1}{N} \sum_{k=0}^{N-1} \exp \left(-\gamma A_{l, m}\left|H_{k}\right|^{2}\right), \\
\zeta_{L E}=\exp \left\{-\mathrm{SNR}_{\mathrm{LE}} A_{l, m}\right\}, \\
\zeta_{D F E}=\exp \left\{-\mathrm{SNR}_{\mathrm{DFE}} A_{l, m}\right\},
\end{array}
$$

the equations (46) and (47) reveal that the monotonic behavior of the logarithmic function allows us to compare the cutoff 
rates by comparing the functions $\zeta_{O F D M}, \zeta_{L E}$ and $\zeta_{D F E}$. In such a case that $\zeta_{a}<\zeta_{b} \Rightarrow R_{0, a}>R_{0, b}$. In order to compare the expressions in (48), (49) and (50), the following function is defined:

$$
\varphi(x)=\exp \left(-A_{l, m} x\right)
$$

and recall the functions $\theta(x)$ and $\phi(x)$ defined by (13) and (14).

Finally, (48), (49) and (50) can be rewritten as:

$$
\begin{array}{r}
\zeta_{O F D M}=\frac{1}{N} \sum_{k=0}^{N-1} \varphi\left(\gamma\left|H_{k}\right|^{2}\right), \\
\zeta_{L E}=\varphi\left(\theta^{-1}\left(\frac{1}{N} \sum_{k=0}^{N-1} \theta\left(\gamma\left|H_{k}\right|^{2}\right)\right)\right), \\
\zeta_{D F E}=\varphi\left(\phi^{-1}\left(\frac{1}{N} \sum_{k=0}^{N-1} \phi\left(\gamma\left|H_{k}\right|^{2}\right)\right)\right),
\end{array}
$$

written in such form, (52), (53) and (54) allow the use of a convex analysis approach in order to establish a comparison between the OFDM and SCCP for any given configuration. In addition, the comparison between the OFDM and SCCP is separated into two different contexts: the comparison between the OFDM and the LE-SCCP, and the comparison between the OFDM and the DFE-SCCP.

In order to accomplish the first comparison, the following is also defined:

$$
\rho(z)=\varphi\left(\theta^{-1}(z)\right) \text {. }
$$

This function allow us to express (52) and (53) as

$$
\begin{gathered}
\zeta_{O F D M}=\frac{1}{N} \sum_{k=0}^{N-1} \rho\left(\theta\left(\gamma\left|H_{k}\right|^{2}\right)\right), \\
\zeta_{L E}=\rho\left(\frac{1}{N} \sum_{k=0}^{N-1} \theta\left(\gamma\left|H_{k}\right|^{2}\right)\right) .
\end{gathered}
$$

The Jensen inequality guarantees that if the function $\rho$ is convex,

$$
\rho\left(\frac{1}{N} \sum_{k=0}^{N-1} \theta\left(\gamma\left|H_{k}\right|^{2}\right)\right) \leq \frac{1}{N} \sum_{k=0}^{N-1} \rho\left(\theta\left(\gamma\left|H_{k}\right|^{2}\right)\right) .
$$

Hence, the convexity of $\rho$ implies $\zeta_{L E} \leq \zeta_{O F D M}$ that is equivalent to state that $R_{0, \mathrm{LE}} \geq R_{0, \mathrm{OFDM}}$.

By definition, a function is convex if and only if its second derivative is non-negative. The second derivative of the function $\rho(z)$ is given by

$$
\frac{d^{2}}{d z^{2}} \rho(z)=A_{l, m} \frac{\rho(z)}{z^{3}}\left(\frac{A_{l, m}}{z}-2\right)
$$

and as $\rho(z)$ is non-negative, the sign of $\frac{d^{2}}{d z^{2}} \rho(z)$ is determined by the function $a(z)$, given by

$$
a(z)=\frac{A_{l, m}}{z}-2 .
$$

However, (56) reveals that the domain values that are important to the cutoff rate calculation are given by $\theta\left(\gamma\left|H_{k}\right|^{2}\right)$. Calculating the function $a(z)$ at these values, we have that:

$$
a\left(\theta\left(\gamma\left|H_{k}\right|^{2}\right)\right)=A_{l, m}\left(\gamma\left|H_{k}\right|^{2}+1\right)-2 .
$$

Moreover, considering a coding rate $r$ and QPSK modulation, with $0<r \leq 1$, if we express $\gamma$ in terms of the SNR per bit, $E_{b} / N_{o}$, we have that:

$$
a\left(\theta\left(\gamma\left|H_{k}\right|^{2}\right)\right)=A_{l, m}\left(2 r \frac{E_{b}}{N_{o}}\left|H_{k}\right|^{2}+1\right)-2 .
$$

In order to assure that $\zeta_{L E} \leq \zeta_{O F D M}$, the channel coefficients have to guarantee that $a\left(\theta\left(\gamma\left|H_{k}\right|^{2}\right)\right) \geq 0$, for a fixed $E_{b} / N_{o}$. Thus, for higher coding rates, it is possible to be less restrictive with regard to the channel coefficients to guarantee the positiveness of (62). This fact points that increasing coding rates represent a more favorable scenario for the LE-SCCP.

A comparison can also be established between the OFDM and the DFE-SCCP schemes in a similar way. In order to do so, the following function is defined:

$$
\vartheta(y)=\varphi\left(\phi^{-1}(y)\right)
$$

Hence, (52) and (54) can be rewritten as

$$
\begin{gathered}
\zeta_{O F D M}=\frac{1}{N} \sum_{k=0}^{N-1} \vartheta\left(\phi\left(\gamma\left|H_{k}\right|^{2}\right)\right), \\
\zeta_{D F E}=\vartheta\left(\frac{1}{N} \sum_{k=0}^{N-1} \phi\left(\gamma\left|H_{k}\right|^{2}\right)\right) .
\end{gathered}
$$

By the analysis of $\frac{d^{2}}{d y^{2}} \vartheta(y)$ expression, we conclude that its sign is given by

$$
b(y)=A_{l, m} \exp (y)-1
$$

and from (64) we can infer that the domain values that are used in the calculation of the cutoff rate are given by $\phi\left(\gamma\left|H_{k}\right|^{2}\right)$. Calculating the function $b(y)$ in these values, we have that:

$$
b\left(\phi\left(\gamma\left|H_{k}\right|^{2}\right)\right)=A_{l, m}\left(\gamma\left|H_{k}\right|^{2}+1\right)-1 .
$$

In terms of $E_{b} / N_{o}$, we can write

$$
b\left(\phi\left(\gamma\left|H_{k}\right|^{2}\right)\right)=A_{l, m}\left(2 r \frac{E_{b}}{N_{o}}\left|H_{k}\right|^{2}+1\right)-1 .
$$

Therefore, the situation is similar to that presented in the comparison between the OFDM and the LE-SCCP. The main difference is that the $b\left(\phi\left(\gamma\left|H_{k}\right|^{2}\right)\right)>a\left(\theta\left(\gamma\left|H_{k}\right|^{2}\right)\right)$. Hence, given a fixed $E_{b} / N_{o}$ and coding rate, the channel class for which (68) is greater than zero is broader than that which guarantees the positiveness of (62).

\section{Monte Carlo approach}

In this subsection, the schemes will be analyzed under different modulations and coding rates scenarios using Monte Carlo simulation for block fading channels.

The schemes will be simulated using the system model described in Section II and Matlab ${ }^{\circledR}$. The simulations will be carried out with the $(133,171)$ convolutional code with rate $r=\frac{1}{2}$. Its punctured version with $r=\frac{3}{4}$ will also be used. Error propagation will be considered in the DFE in order to show more realistic performance results. We considered that the OFDM is implemented with $N=512$ subcarriers and that the block length in the SCCP is equal to $N=512$ symbols. 


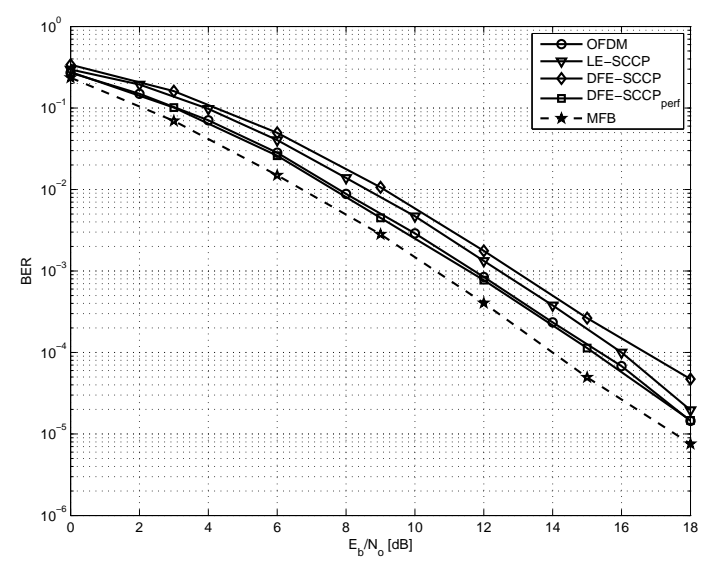

Fig. 4. Performance comparison for the channel with transfer function given by (69) with QPSK modulation and $r=\frac{1}{2}$.

For all simulations, we have used a block fading channel with transfer function:

$$
H(z)=h_{0}+h_{1} z^{-1}+h_{2} z^{-2}
$$

where the coefficients $h_{k}, k=0,1,2$ are complex Gaussian random variables with zero mean and variance $\sigma_{h_{k}}^{2}=1 / 3$.

Since a frequency selective channel is used, error bursts can be expected in the OFDM and colored noise in the output of the SCCP equalizer. However, the used convolutional code is designed for random errors, and thereby an interleaver is necessary to randomize the errors or to whiten the noise. As pointed out by [38], different interleaver configurations can lead to significant differences in the performance of the schemes. In [38], through extensive simulations, different types of interleavers were analyzed for block fading channels and it was shown that the matrix interleaver is a suitable choice for both schemes if the number of columns and rows are appropriately chosen for a given channel and modulation scheme. Therefore, for the following simulations, we have adopted the matrix interleaver parameters accordingly to [38].

The following simulations will compare the OFDM and the SCCP for different coding rates, modulation orders and spectral efficiencies.

1) Coding rate: The impact of different coding rates for the comparison between the schemes will be investigated. In order to do so, QPSK modulation will be considered. The results obtained for the simulation with $r=\frac{1}{2}$ and $\frac{3}{4}$ are shown in Figs. 4 and 5, respectively. Considering $r=\frac{1}{2}$, the OFDM presents the same performance of the perfect DFE-SCCP. In addition, the gap between the OFDM or the perfect DFE-SCCP and the matched filter bound (MFB) is not superior to $1 \mathrm{~dB}$. This means that even a more powerful equalization scheme, such as turbo equalization, would not provide a significant gain over the OFDM.

Another important point is that the error propagation degrades the DFE-SCCP in such a way that its performance is even inferior to that of the LE-SCCP. The error propagation phenomenon with coded schemes was analyzed in [39], where it was shown situations in which the LE-SCCP provides a better performance than the DFE-SCCP under certain conditions. Although there are some techniques that are able to mitigate

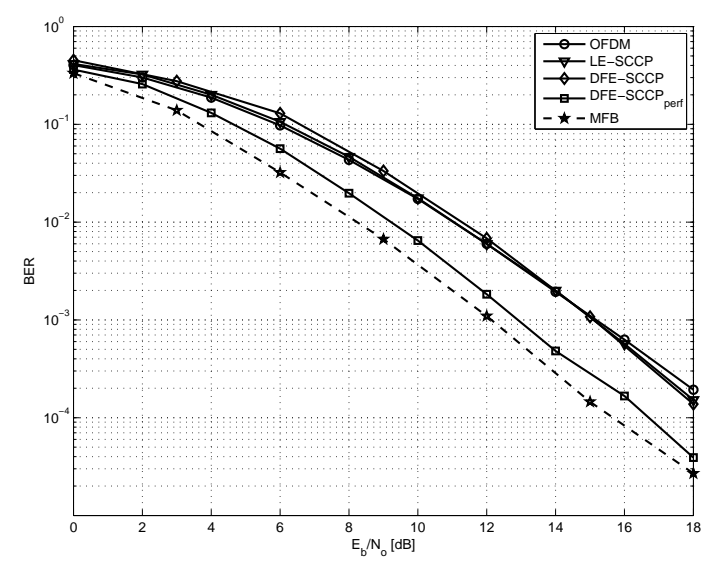

Fig. 5. Performance comparison for the channel with transfer function given by (69) with QPSK modulation and $r=\frac{3}{4}$.

this effect (e.g., [40]) and to approximate the DFE-SCCP performance to the perfect DFE-SCCP, there is an additional computational cost associated to them. Therefore, even if the error propagation mitigation technique provides a performance approximately equal to the perfect DFE-SCCP, it would be costlier than the OFDM. Thus, for this modulation and coding scheme, the OFDM provides a better solution than the SCCP scheme.

Concerning the coding rate $r=3 / 4$, we can note a performance degradation of the OFDM. In this case, its performance is equivalent to the LE-SCCP, whereas for $r=1 / 2$, its performance is equivalent to the perfect DFE-SCCP. This performance degradation of the OFDM for higher coding rates is not unexpected, since we have already stated that when an arbitrary high coding rate is used, which takes us closer to the uncoded case, the SCCP presents a superior performance for any channel configuration when the QPSK modulation is employed.

Such BER results are corroborated by the cutoff analysis. For each coding rate, the probability that the transmitted spectral efficiency is above the cutoff rate is evaluated through Monte Carlo simulation, i.e., for each channel realization the cutoff rate given by (46) and (47) is calculated and compared to the given spectral efficiency. Such probability will be referred as the cutoff rate outage probability. The cutoff rate outage probability is estimated through Monte Carlo simulation and the results are shown in Fig. 6 for $r=1 / 2,3 / 4$ and $9 / 10$. From Fig. 6, we observe a degradation in the performance of the OFDM in comparison to the SCCP with increasing coding rate. For $r=1 / 2$, the cutoff rate outage probability associated to the OFDM is slightly lower than the one associated to the LE-SCCP. For higher coding rates, the LE-SCCP outperforms the OFDM. Concerning the DFESCCP, it outperforms all the other techniques for the simulated coding rates.

2) Modulation order: The modulation order is also an important parameter in the comparison between the OFDM and the SCCP. In the uncoded case, it was shown that the SCCP always excels the OFDM when QPSK modulation is employed, but this statement is not true for higher order modulations. In order to infer if this performance degradation 

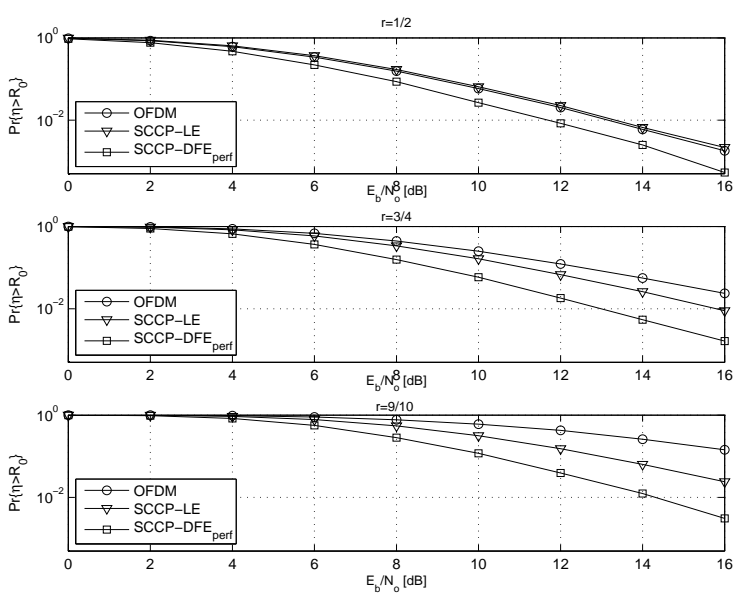

Fig. 6. Cutoff rate outage for QPSK modulation and channel with transfer function given by (69).

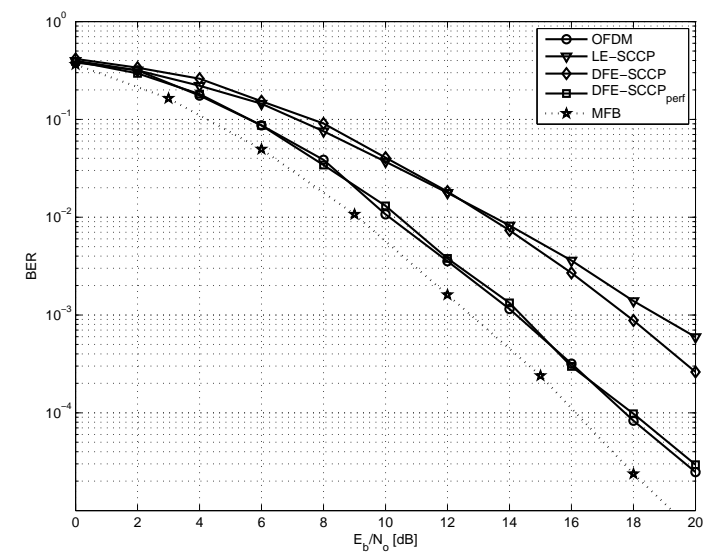

Fig. 7. Performance comparison for the channel with transfer function given by (69) with 16-QAM and $r=\frac{1}{2}$.

presented by the SCCP with increasing modulation order is also observed in the coded context, BER simulations considering QPSK, 16-QAM and 64-QAM modulations and $r=1 / 2$ are analyzed. The results are shown in Figs. 4, 7 and 8, respectively.

From these results, we can infer that the LE-SCCP presents a performance degradation when compared to the OFDM for higher order modulations. Considering QPSK modulation, the

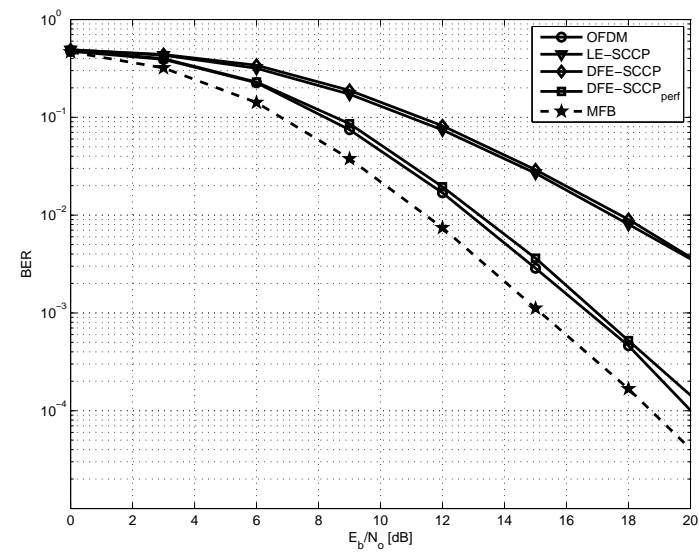

Fig. 8. Performance comparison for the channel with transfer function given by (69) with 64-QAM and $r=\frac{1}{2}$.

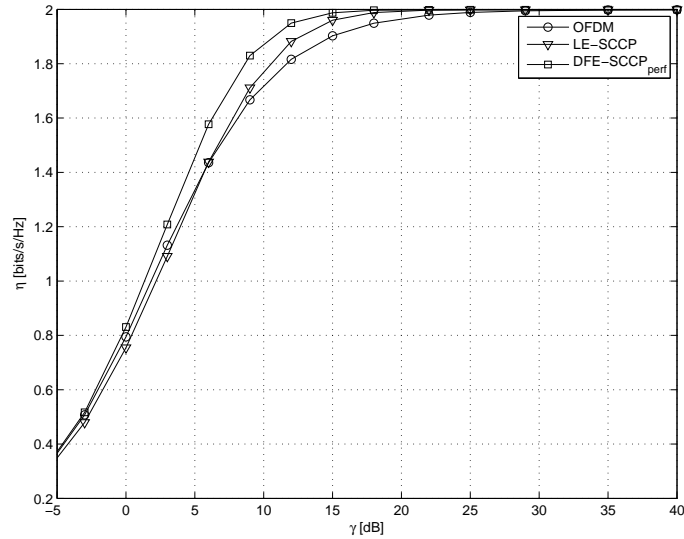

Fig. 9. Capacity for the channel with transfer function given by (69) for QPSK modulation.

performance gap between the OFDM and the LE-SCCP is of approximately $2 \mathrm{~dB}$, for the 16-QAM modulation, this gap increases to $5 \mathrm{~dB}$ and considering 64-QAM, the gap is equal to $6 \mathrm{~dB}$. Such performance degradation is not observed for the perfect DFE-SCCP and its advantage in the uncoded case for QPSK and 16-QAM disappears.

Next, in order to show the sensitiveness to the modulation order for other coding rates, the channel capacity is obtained. For each channel realization, the theoretical channel capacity is evaluated using (39) and (40) and the average capacity is estimated with Monte Carlo simulation. The results for the QPSK modulation are shown in Fig. 9 and the results for several modulations order are shown in Fig. 10. For QPSK modulation, we can observe that the schemes present approximately the same performance for $r=1 / 2$ and spectral efficiency $\eta$, defined by $r \log _{2}(M)$, equal to 1 . For lower coding rates, the OFDM slightly outperforms the LE-SCCP and for $r \rightarrow 1$, the LE-SCCP slightly surpasses the OFDM. This fact is expected once the OFDM is highly dependent on the coding rate. Considering 16-QAM, with $r=1 / 2(\eta=2)$, the OFDM outperforms the LE-SCCP by approximately 1.2 $\mathrm{dB}$. In this case, the LE-SCCP will only outperform the OFDM for a spectral efficiency close to $\eta=3.8$, which corresponds to $r=0.95$. Finally, for 64-QAM and $r=1 / 2$, the OFDM surpasses the LE-SCCP by $1.7 \mathrm{~dB}$ and the LE-SCCP will surpass the OFDM only for $\eta=5.93$, which corresponds to $r=0.98$. Therefore, the capacity analysis also shows a performance degradation of the LE-SCCP with increasing modulation order.

Concerning the perfect DFE-SCCP, from Fig. 10, it presents a capacity equal to or superior to the OFDM depending on the coding rate. Considering $r=1 / 2$, it presents the same capacity as the OFDM for the analyzed modulation schemes. Although, for increasing coding rates, the DFESCCP surpasses the OFDM capacity. This result corroborates the BER simulations.

3) Spectral efficiency: It was previously shown that the OFDM undergoes performance degradation as the coding rate increases. In addition, it was observed that the LE-SCCP scheme presents performance degradation for higher order modulations. In the following, both schemes will be compared 


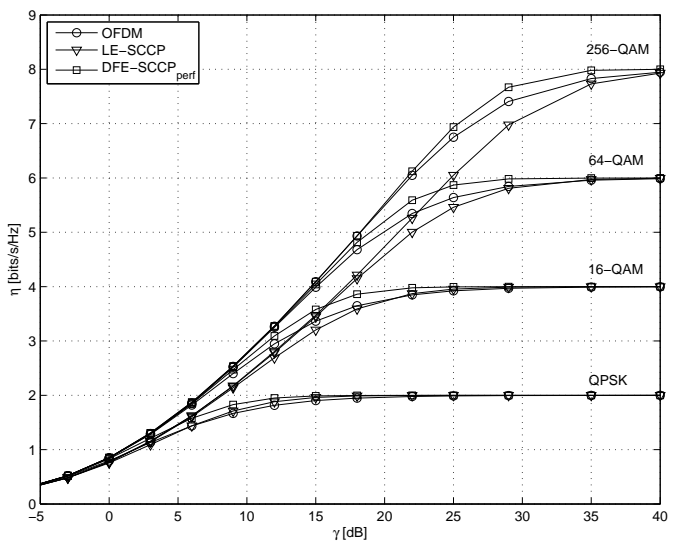

Fig. 10. Capacity for the channel with transfer function given by (69) for several modulations order.

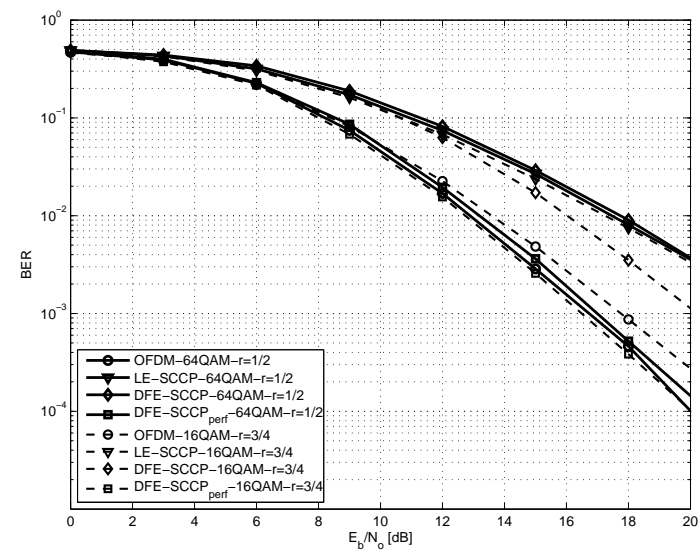

Fig. 11. Performance comparison for a fixed spectral efficiency, $\eta=3$ and for the channel with transfer function given by (69).

changing the modulation order and the coding rate, while the spectral efficiency is fixed.

Therefore, if we employ 64-QAM with $r=\frac{1}{2}$, the system spectral efficiency will be given by $\eta=3$. This is the same spectral efficiency of a system employing 16-QAM and $r=\frac{3}{4}$. In Fig. 11, a performance comparison between the schemes in these two modulations and rates configurations is shown. For the OFDM, the 64-QAM with $r=\frac{1}{2}$ can be observed to provide better results than the 16-QAM with $r=\frac{3}{4}$. By contrast, for the SCCP, the 16-QAM with $r=\frac{3}{4}$ is marginally a better option.

It is important to point out that the OFDM can achieve these BER results and the perfect DFE is just an idealization.

In addition to the BER simulations, the Shannon capacity outage probability for these two modulation and coding schemes could be analyzed. The results are shown in Fig. 12. Comparing these results with the ones obtained with the BER simulation given in Fig. 11, we can see that they are in accordance: in both cases the OFDM performs equal to the DFE-SCCP for the best modulation and coding scheme.

The difference in the results provided by the BER and outage analysis is that for the SCCP, the modulation and coding scheme with $16-\mathrm{QAM}$ and $r=3 / 4$ provides slightly better BER results than the 64-QAM with $r=1 / 2$. The opposite occurs for the outage probability. Such small divergence

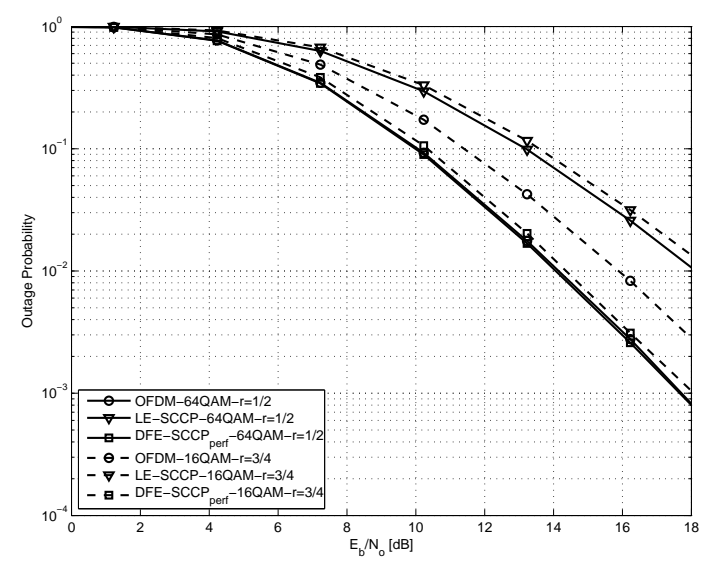

Fig. 12. Outage probability for spectral efficiency fixed in $\eta=3$.

can be expected once the outage probability considers some unrealistic assumptions, such as that the ISI is Gaussian.

\section{Conclusions}

In this article we have established a performance comparison between the OFDM and the SCCP schemes under different contexts when no channel information is present on the transmitter side and for frequency selective block fading channels. Although the SCCP outperforms the OFDM for any channel configuration when QPSK modulation is employed in the uncoded scenario, the use of good error correcting codes, i.e. codes with long constraint lengths and low rates, tend to leverage the OFDM, making the performance differences much more subtle. In order to provide a deeper insight on this, we have revisited some previous results in the literature and provided new ones through the use of Monte Carlo simulations, Shannon capacity and a convex framework analysis in which the effective SNR and the cutoff rate were employed to compare the schemes performance. Based on the results obtained, it was shown that if adequate coding rates and modulation schemes were chosen, the OFDM will present the same performance as the best analyzed single carrier technique, i.e., the perfect DFE-SCCP. Since the latter is just an idealization, we can affirm that the OFDM is the best choice among the analyzed techniques for frequency selective block fading channels.

\section{REFERENCES}

[1] R. W. Chang, "Synthesis of band-limited orthogonal signals for multichannel data transmission," Bell Sys. Tech. J., vol. 45, 1966.

[2] H. Sari, G. Karam, and I. Jeanclaude, "Transmission techniques for digital terrestrial TV broadcasting," IEEE Communications Magazine, vol. 33, no. 2, pp. 100-109, Feb. 1995.

[3] S.K. Wilson and J.M. Cioffi, "A comparison of a single-carrier system using a DFE and a coded OFDM system in a broadcast rayleigh-fading channel," Process. of IEEE Int. Symp. on Information Theory, 1995, pp. 335-, Sep 1995.

[4] A. Peled and A. Ruiz, "Frequency domain data transmission using reduced computational complexity algorithms," in ICASSP, 1980, pp. 964-967.

[5] Y. (G.) Li and G. Stübe, Orthogonal Frequency Division Multiplexing for Wireless Communications, Springer, 2006.

[6] Haitao Li, Xiaojun Yuan, Xiaokang Lin, and Li Ping, "On water-filling precoding for coded single-carrier systems," Communications Letters, IEEE, vol. 13, no. 1, pp. $34-36,2009$. 
[7] T.J. Willink and P.H. Wittke, "Optimization and performance evaluation of multicarrier transmission," Information Theory, IEEE Transactions on, vol. 43, no. 2, pp. $426-440$, Mar. 1997.

[8] J. Louveaux, L. Vandendorpe, and T. Sartenaer, "Cyclic prefixed single carrier and multicarrier transmission: bit rate comparison," Communications Letters, IEEE, vol. 7, no. 4, pp. 180 - 182, 2003.

[9] Austin M. E., "Decision feedback equalization for digital communication over dispersive channels," MIT Research Laboratory of Electronics Technical Report, vol. 461, Aug.

[10] N. Benvenuto and S. Tomasin, "On the comparison between OFDM and single carrier modulation with a DFE using a frequency-domain feedforward filter," IEEE Trans. On Communications, vol. 50, no. 6, June 2002.

[11] H. Harashima and H. Miyakawa, "Matched-transmission technique for channels with intersymbol interference," Communications, IEEE Transactions on, vol. 20, no. 4, pp. 774 - 780, Aug. 1972.

[12] J.M. Cioffi, G.P. Dudevoir, M. Vedat Eyuboglu, and Jr. Forney, G.D., "MMSE decision-feedback equalizers and coding. i. equalization results," Communications, IEEE Transactions on, vol. 43, no. 10, pp. $2582-2594$, Oct. 1995

[13] J.M. Cioffi, G.P. Dudevoir, M.V. Eyuboglu, and Jr. Forney, G.D., "MMSE decision-feedback equalizers and coding. ii. coding results," Communications, IEEE Transactions on, vol. 43, no. 10, pp. 2595 2604, Oct. 1995.

[14] D. Falconer, S. L. Ariyavisitakul, A. Benjamin-Seeyar, and B. Edison, "Frequency domain equalization for single-carrier broadband wireless systems," IEEE Communications Magazine, vol. 40, pp. 58-66, April 2002.

[15] Yuan-Pei Lin and See-May Phoong, "BER minimized OFDM systems with channel independent precoders," Signal Processing, IEEE Transactions on, vol. 51, no. 9, pp. 2369-2380, Sept. 2003

[16] V. Aue, G. P. Fettweis, and R. Valenzuela, "A comparison of the performance of linearly equalized single carrier and coded OFDM over frequency selective fading channels using the random coding technique," in Proc. Int. Conf. Communications (ICC), Atlanta, 1998, vol. 2, pp. 753-757.

[17] L. Van der Perre, J. Tubbax, F. Horlin, and H. De Man, "A singlecarrier/OFDM comparison for broadband wireless communication," in Acoustics, Speech, and Signal Processing, 2004. Proceedings. (ICASSP '04). IEEE International Conference on, May 2004, vol. 2, pp. ii-32932 vol. 2 .

[18] Zhengdao Wang, Xiaoli Ma, and G.B. Giannakis, "OFDM or singlecarrier block transmissions?," Communications, IEEE Transactions on, vol. 52, no. 3, pp. 380-394, March 2004.

[19] Yuan-Pei Lin and See-May Phoong, "MMSE OFDM and prefixed single carrier systems: BER analysis," Acoustics, Speech, and Signal Processing, 2003. Proceedings. (ICASSP '03). 2003 IEEE International Conference on, vol. 4, pp. IV-229-32 vol.4, April 2003.

[20] B. Devillers, J. Louveaux, and L. Vandendorpe, "About the diversity in cyclic prefixed single-carrier systems," Physical Communication, Elsevier, vol. 1, pp. 266-276, Dec. 2008.

[21] I. M. Jacobs J. Wozencraft, Principles of Communication Engineering, Wiley, 1965

[22] E. Westman, "Calibration and evaluation of the exponential effective SINR mapping (EESM) in 802.16," M.S. thesis, KTH Electrical Engineering, 2007.

[23] M. Salehi J. Proakis, Digital Communications, Mc Graw-Hill, 5 edition, 2008.

[24] R. Lopez-Valcarce, "Realizable linear and decision feedback equalizers: properties and connections," Signal Processing, IEEE Transactions on, vol. 52, no. 3, pp. 757-773, March 2004.

[25] H. Witschnig, T. Mayer, A. Springer, A. Koppler, L. Maurer, M. Huemer, and R. Weigel, "A different look on cyclic prefix for SC/FDE," in Personal, Indoor and Mobile Radio Communications, 2002. The 13th IEEE International Symposium on, 2002, vol. 2, pp. 824 - 828 vol.2.

[26] G. Pólya G. H. Hardy, J. E. Littewood, Inequalities, Cambridge, 1952.

[27] J. Salz, "Optimum mean-square decision feedback equalization," Bell Syst. Tech. J., vol. 52, pp. 1341-1373, Oct. 1973.

[28] A. S. Paula and C. Panazio, "An uncoded BER comparison between DFE-SCCP and OFDM using a convex analysis framework," in ISCAS, 2011.

[29] Y. Nasser, J.F. Helard, and M. Crussiere, "Bit error rate prediction of coded MIMO-OFDM systems," in Signal Processing Advances in Wireless Communications, 2008. SPAWC 2008. IEEE 9th Workshop on, July 2008 , pp. 181-185.
[30] H. Song, R. Kwan, and J. Zhang, "On statistical characterization of EESM effective SNR over frequency selective channels," Wireless Communications, IEEE Transactions on, vol. 8, no. 8, pp. $3955-3960$, august 2009.

[31] Hui Song, Raymond Kwan, and Jie Zhang, "Approximations of EESM effective SNR distribution," Communications, IEEE Transactions on, vol. 59, no. 2, pp. $603-612,2011$.

[32] T.L. Jensen, S. Kant, J. Wehinger, and B.H. Fleury, "Mutual information metrics for fast link adaptation in IEEE 802.11n," in Communications, 2008. ICC '08. IEEE International Conference on, May 2008, pp. 4910 $-4915$.

[33] Xiang Chen, Zesong Fei, Wei Sun, and Jingming Kuang, "Link adaptation of rate-compatible LDPC coded OFDM system based on minus exponential effective-SNR mapping link quality model," in Wireless Communications, Networking and Mobile Computing, 2008. WiCOM '08. 4th International Conference on, 2008, pp. 1 -5.

[34] Y. Nasser, M. Des Noes, L. Ros, and G. Jourdain, "On the system level prediction of joint time frequency spreading systems with carrier phase noise," Communications, IEEE Transactions on, vol. 58, no. 3, pp. 839 $-850,2010$.

[35] R. Sandanalakshmi, T.G. Palanivelu, and K. Manivannan, "Effective SNR mapping for link error prediction in OFDM based systems," in Information and Communication Technology in Electrical Sciences (ICTES 2007), 2007. ICTES. IET-UK International Conference on, 2007, pp. $684-687$.

[36] P. Viswanath D. Tse, Fundamentals of Wireless Communication, Cambridge, 2005

[37] Joy A. Thomas Thomas M. Cover, Elements of Information Theory, Wiley, 2006.

[38] A.S. Paula, "Um estudo comparativo entre os sistemas OFDM e SCCP," M.S. thesis, Escola Politecnica, University of Sao Paulo, 2010.

[39] M.V. Eyuboglu, "Detection of coded modulation signals on linear, severely distorted channels using decision-feedback noise prediction with interleaving," Communications, IEEE Transactions on, vol. 36 , no. 4, pp. $401-409$, Apr. 1988.

[40] C. M. Panazio and J. M. T. Romano, "Performance of joint spacetime equalization and decoding techniques for wireless systems," in International Telecommunications Symposium-ITS, Natal, Brazil, 2002.

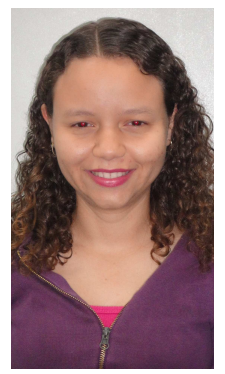

Amanda de Paula received the B.Sc. degree from Federal University of Pernambuco (UFPE) in 2008 and the M.Sc. degree from Escola Politécnica of the University of São Paulo (EPUSP) in 2010, both in electrical engineering. She is currently pursuing the $\mathrm{Ph} . \mathrm{D}$. degree also in electrical engineering at Escola Politécnica of the University of São Paulo (EPUSP). Her research interests include multicarrier modulations, cognitive radio, detection and estimation theory.

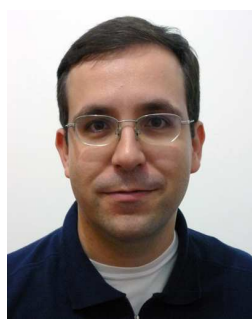

Cristiano Panazio received a B.Sc. and an M.Sc. degree in electrical engineering from the State University of Campinas (UNICAMP), Brazil, in 1999 and 2001, respectively. He received his Ph.D. in 2005, also in electrical engineering, from the Conservatoire National des Arts Métiers (CNAM), Paris, France. In 2006, he became assistant professor at Escola Politécnica of the University of São Paulo. $\mathrm{He}$ is member of the Brazilian Telecommunications Society (SBrT) since 1999. His research interests include equalization, multicarrier modulations and spread spectrum techniques. 